\title{
Corticofugal Influences of S1 Cortex on Ventrobasal Thalamic Neurons in the Awake Rat
}

\author{
Bin Yuan, ${ }^{*, 1}$ Thomas J. Morrow, $\dagger$ and Kenneth L. Casey $\dagger$ \\ "Xian Medical University, People’s Republic of China, Departments of \$Neurology and †Physiology, University of \\ Michigan, and Neurology Research Laboratories (Neurophysiology), Veterans Administration Medical Center, Ann \\ Arbor, Michigan 48105
}

\begin{abstract}
Corticofugal influences on the responses of 39 ventrobasal (VB) thalamic neurons to repetitive stimuli were studied in awake rats by focally suppressing the evoked and spontaneous electrocortical activity of the primary (S1) somatosensory cortex with magnesium or lidocaine plus magnesium. Suppression of the $\mathrm{S} 1$ cortex reduced the number of spikes discharged by $19(66 \%)$ of 29 VB units in response to each of 25 electrical stimuli delivered to the medial lemniscus; 9 units were unaffected and 1 showed an increased response to $1-10 \mathrm{~Hz}$ stimuli. The responses of 6 $(38 \%)$ of $16 \mathrm{VB}$ units to electrical somatic stimuli were also reduced following S1 cortical suppression; 9 units were unaffected and 1 showed an increased response to 20-40 Hz stimuli. A comparison of the reduced responsiveness of 5 units studied during medial lemniscal and somatic stimulation did not reveal any additional response attenuation attributable to subthalamic corticofugal influences. We conclude that, in the awake rat, somatosensory transmission to VB thalamic neurons is primarily facilitated by $S 1$ corticothalamic neurons.
\end{abstract}

In previous work (Yuan et al., 1985), we have shown that, in the anesthetized rat, focal suppression of the $S 1$ cortex decreases the excitability of ventrobasal (VB) thalamic neurons, as tested by their responses to repetitive somatic or medial lemniscal stimuli delivered at various frequencies. A comparison of the effect of cortical suppression on the responses of VB neurons to somatic and medial lemniscal inputs suggested that excitatory corticothalamic neurons mediated a significant fraction of the corticofugal influence.

Because general anesthesia affects the activity of neurons in the cerebral cortex, we wished to determine if focal suppression of $\mathrm{S} 1$ corticofugal influences in the awake preparation has effects that are substantially different from those seen in the anesthetized animal. For example, excitatory or inhibitory corticofugal effects on the dorsal column nuclei or the dorsal horn could be increased relative to facilitatory corticothalamic activity. The suppression of $\mathrm{S} 1$ cortex would then be expected to have a greater effect when VB neurons were activated cutaneously than when medial lemniscal stimulation was used. In the series of experiments reported here, we present evidence that, in the awake as in the anesthetized rat, S1 corticothalamic neurons facilitate somatosensory transmission in the VB thalamus and that, in the awake rat, corticothalamic facilitation appears to predominate over other inhibitory corticofugal influences.

\footnotetext{
Received Dec. 6, 1985; rcvised Apr. 14, 1986; accepted Apr. 17, 1986.
}

This work was supported by the Veterans Administration. We thank Patricia Morris for valuable technical assistance.

Correspondence should be addressed to Kennellı L. Casey, M.D., Neurology Service (127), VA Medical Center, 2215 Fuller Road, Ann Arbor, MI 48105.

' On leave; Fellow, Ministry of Higher Education.

$0270-6474 / 86 / 123611-07 \$ 02.00 / 0$

\section{Materials and Methods}

Fourteen male albino rats, weighing $350-550 \mathrm{gm}$, were used in this study. Most of the rats were adapted to a restraining device (see below) for 2-3 d before being selected for surgery. Each rat was anesthetized (chloral hydrate, $40 \mathrm{mg} / 100 \mathrm{gm}$ ), and a plastic cylinder, $6 \mathrm{~mm}$ in diameter and $8 \mathrm{~mm}$ in height, was implanted in the skull overlying the intact dura of the right S1 cortex (coordinates: AP 2.5 to -3.0, ML 2.07.5; Pelligrino et al., 1979). The cylinder was capped tightly and a pledget of Gelfoam soaked with ophthalmic ointment containing bacitracin, neomycin, and polymyxin (Byk-Gulden, Melville, NY) was put on the dura to prevent infection. During experiments, the antibiotic pledget was removed and the dura was cleaned with normal saline so that solutions of drugs could be applied to the surface of the dura. Two electrodes made of Teflon-coated stainless steel wire were implanted on the dura over the cortical forepaw and facial regions. These electrodes were used for monopolar recording of cortical evoked potentials, bipolar recording of the surface electrocorticographic $(E C O G)$ activity of the $S 1$ cortex, and, in some cases, bipolar stimulation of the $\mathrm{S} 1$ cortex. One or 2 pairs of stimulating electrodes were implanted in the medial lemniscus in the right pons, and in 2 rats a pair of electrodes with a tip separation of about $1 \mathrm{~mm}$ was implanted in the ipsilateral cerebral peduncle to record corticofugal discharges induced by stimulation of the $S 1$ cortex. A microdrive system (Morrow, 1980) was implanted in the left skull so microelectrodes could be inserted at an angle through the left hemisphere toward the VB thalamus on the right side. We found it was necessary to modify the microdrive system to eliminate twisting of the microelectrode and thus prevent tissue trauma, which was seen along the angular trajectory of some of the earlier microelectrode tracks. Satisfactory unit isolation, recording stability, and stereotaxic placement were not achieved in these animals.

The animals were allowed to recover from the surgery for at least 2 $\mathrm{d}$ before recording was attempted. Each rat was briefly restrained by inhalation of halothane and placed in a flexible, molded cast restraint with the head, limbs, tail, abdomen, and parts of the back exposed. Unit recording did not begin until at least $1 \mathrm{hr}$ after halothane inhalation had ceased. During the periods of unit recording, the rats appeared alert, made infrequent brief, slight movements, and had low-voltage, desynchronized electrocortical activity.

While the stainless steel microelectrode (8-12 m 2 impedance at 100 $\mathrm{Hz}$ ) was lowered toward the VB thalamus, the contralateral body surface was brushed, touched, or pressed to activate neurons. VB neurons were identified by their small contralateral receptive fields and short-latency responses to medial lemniscal or peripheral stimulation. Single units were isolated by a window discriminator on the basis of spike amplitude. Only those units that responded to innocuous mechanical stimuli of the skin were studied.

The values of medial lemniscal stimulation $(0.1 \mathrm{msec}$, usually less than $100 \mu \mathrm{A}$ ) were set between threshold and $2 \times$ threshold to obtain a high unit response probability at a stimulation rate of $1 \mathrm{~Hz}$ and no behavioral response at a frequency of $50 \mathrm{~Hz}$. Receptive fields on the body or limbs could occasionally be stimulated electrically without disturbing the animal. Sharp bipolar needle electrodes could be inserted subcutaneously without eliciting a behavioral response any morc vigorous than that accompanying a drug injection; and at the just-suprathreshold parameters used in this study $(0.1 \mathrm{msec}$, less than $0.5 \mathrm{~mA})$, the rats remained quiet throughout the stimulus period. 
Figure 1. Effect of lidocaine and magnesium sulfate on ECoG of the $\mathrm{S} 1$ cortex of an awake rat. Bipolar recording from intact dura. $A$, Control. $B$, three minutes after applying $5 \%$ lidocaine to the dura. Paroxysmal bursts accompanied by contralateral limb movements. Three $(C)$ and $20(D) \mathrm{min}$ after adding $2 \%$ magnesium sulfate to the dura. Paroxysmal bursting has ceased and background ECoG activity has decreased in frequency and amplitude. $E$, Twenty minutes after washing dura with sterile saline. Time marks, 1 sec.

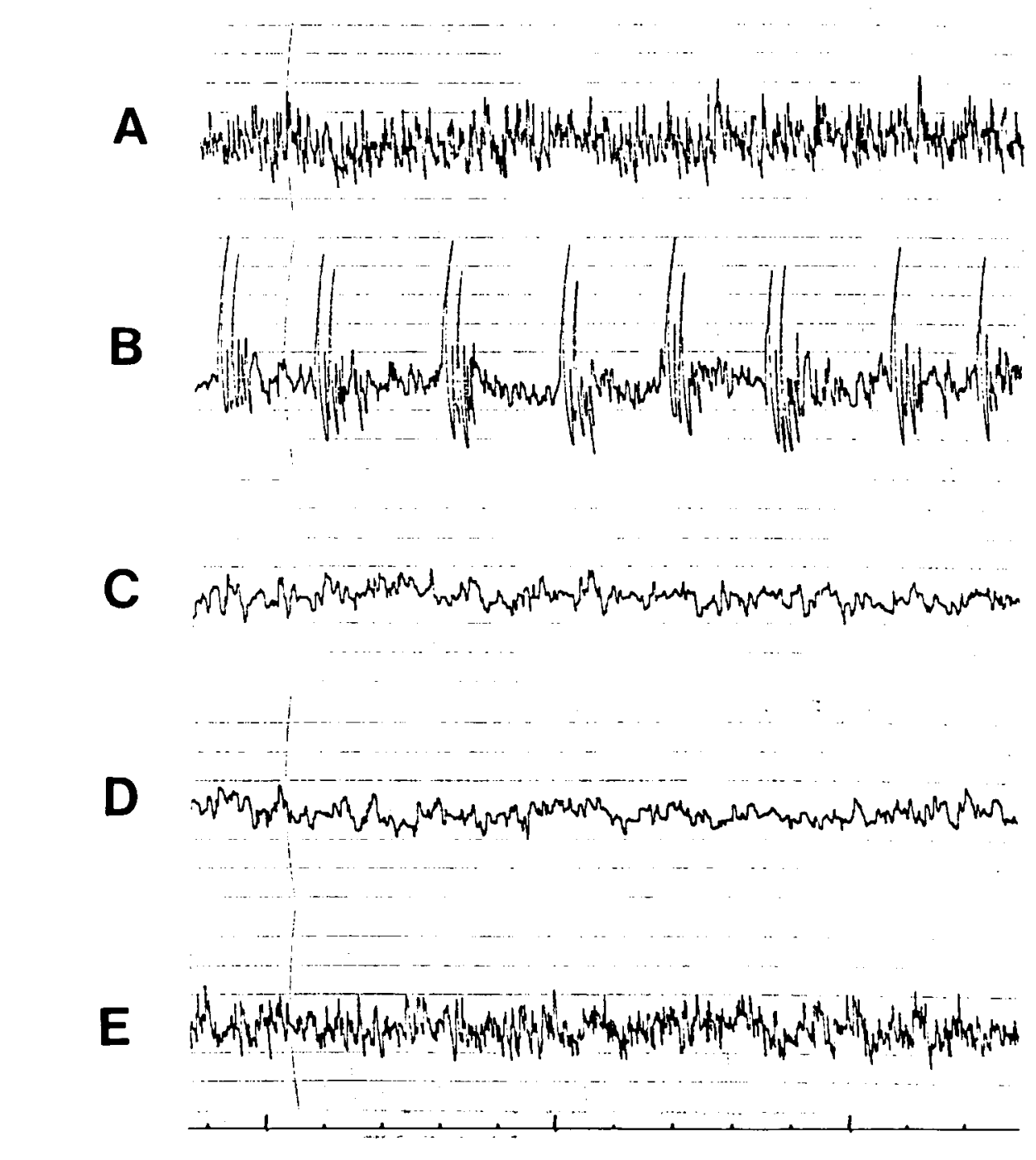

long the track by passing anodal DC current ( $20 \mu \mathrm{A}$ for $20 \mathrm{sec}$ ) through the microelectrode. During deep anesthesia produced with chloral hydrate, the animal was perfused through the heart with $10 \%$ formalin in normal saline. Serial sections were cut at $50 \mu \mathrm{M}$ in the coronal stereotaxic plane and stained with cresyl violet. Electrode tracks were reconstructed from drawings of projected images of the sections.

\section{Results}

\section{Effects of magnesium sulfate on spontaneous and evoked cortical} activity

Six rats were used exclusively to test the effect of lidocaine and magnesium sulfate on cortical activity. In our previous study of anesthetized rats, we showed that $20 \%$ lidocaine applied by pledget to the dura produced focal and reversible suppression of S1 ECoG activity, evoked responses, and corticofugal discharges (Yuan et al., 1985). In the present study of awake rats, we found that topical lidocaine at concentrations of less than $1 \%$ had little effect, but concentrations of $1 \%$ or more produced focal seizures. However, the convulsant effect of lidocaine could be completely eliminated and focal depression of ECoG activity achieved by the addition of $2 \%$ magnesium sulfate, as shown in Figure 1 . We found that $2 \%$ lidocaine in $2 \%$ magnesium sulfate effectively and reversibly suppressed S1 ECoG activity, evoked responses, and corticofugal discharges recorded from the ipsilateral cerebral peduncle. We used this method for S1 suppression in 18 experiments before determining that $4 \%$ mag- 
A
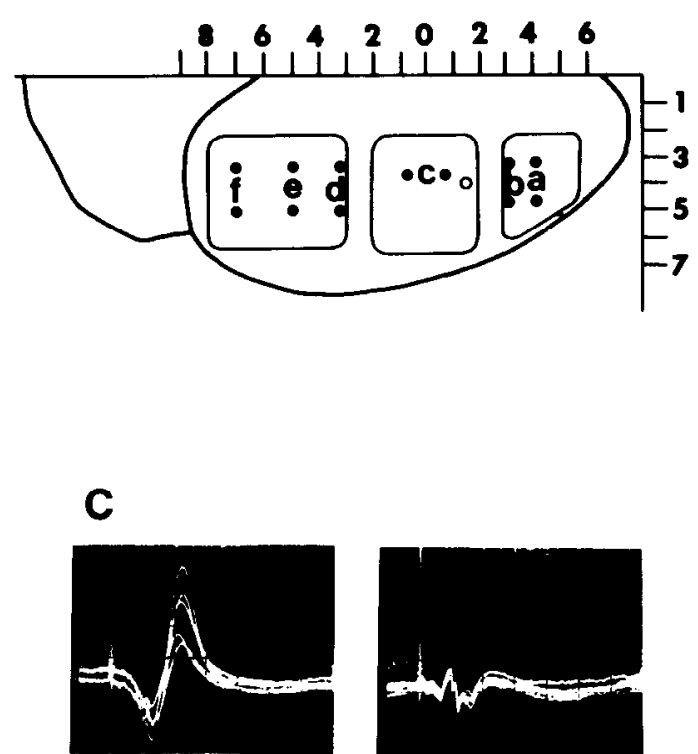

B

a

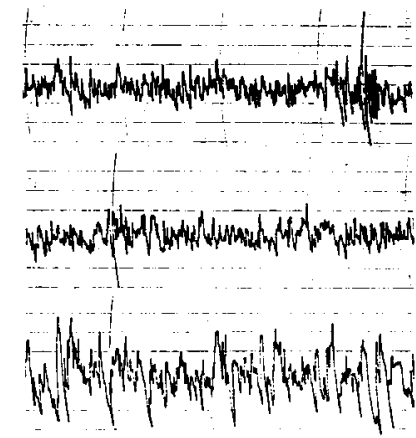

d

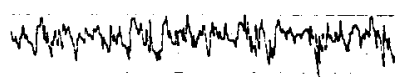

e

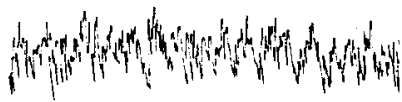

f

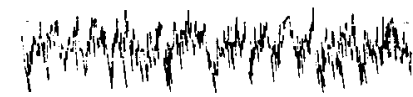

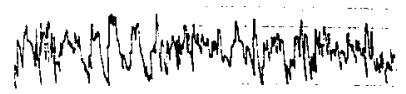
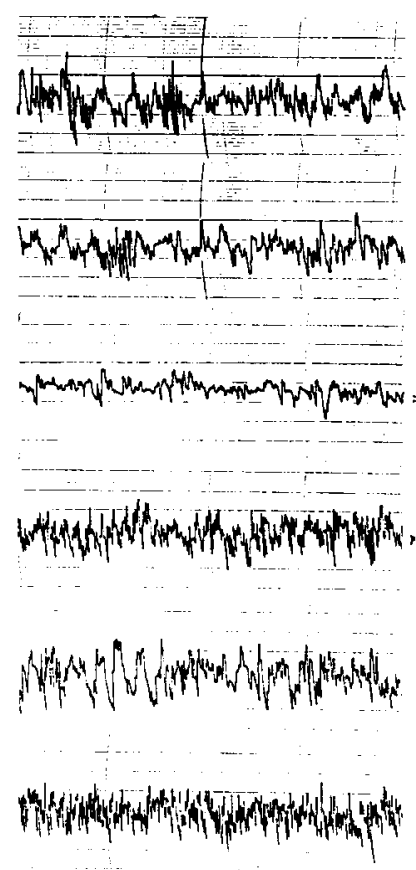

Figure 2. Focal depression of ECoG (bipolar recording; filled circles in A) and forepaw-evoked potentials (monopolar recording; open circles in $A$ ) recorded in chloral hydrate-anesthetized rat from 6 sites $(a-f)$ on the dura exposed by 3 separate craniotomies. Stereotaxic coordinates shown on diagram in $A$. B, ECoG recorded from each of the sites shown in $A$ before (left) and 20 min after (right) applying $4 \%$ magnesium sulfate to the dura at recording site $c$. Note marked reduction in $\mathrm{ECoG}$ amplitude seen only at $c$. $C$, Concurrent recording of reduction in amplitude of S1 forepaw-evoked potential.

nesium sulfate alone was also effective, as shown in Figure 2. In both awake and anesthetized rats, application of $2-4 \%$ magnesium sulfate by pledget to the dura reduced S1 ECoG activity and evoked potential responses to medial lemniscal stimulation within 3-5 min.

Peak effects appeared in approximately $20 \mathrm{~min}$ and could be reversed within an hour after removing the pledget and washing the dura with saline. As determined by ECoG recording, the suppressed area did not extend more than $1 \mathrm{~mm}$ in any direction beyond the borders of the rat S1 cortex, as defined by Chapin and Lin (1984). We saw no evidence for the spreading depression of cortex that is typically produced by application of potassium (Leao, 1944). Accordingly, we used magnesium sulfate atone for focal cortical suppression in the last 21 experiments.

Although ECoG activity was not completely eliminated, the amplitude of the $S 1$ responsc to medial lcmniscal stimulation was markedly reduced (Fig. 2). This effect could be measured conveniently and accurately, and was therefore used for measuring and monitoring the degree of $\mathrm{S} 1$ suppression in the awake rat. Cortical suppression was defined as a reduction of the $S 1$ evoked response amplitudes to less than $50 \%$ of the pretreatment value.

\section{Effect of $S I$ cortical suppression on the responsiveness of $V B$ thalamic somatosensory neurons}

A total of 39 single VB units were isolated and tested before and 20 min after $\mathrm{S} 1$ cortical suppression in 8 awake rats. The histologically reconstructed recording sites are shown in Figure 3. All units were spontaneously active and were responsive to innocuous mechanical stimulation of the skin, hair, or vibrissae within small contralateral receptive fields.

\section{Medial lemniscal stimulation}

Twenty-nine VB neurons responded to single medial lemniscal stimuli with 1 or 2 spikes at latencies of $1-2$ msec and main- tained a relatively high response probability throughout the range of stimulation frequencies $(1-50 \mathrm{~Hz})$ tested. Suppression of the S1 cortex was produced with $2-4 \%$ lidocaine containing $2 \%$ magnesium sulfate while recording 16 units, and with $2-4 \%$ magnesium sulfate alone while studying 13 other units. The results were similar with either method. After the S1 cortex was suppressed, 19 units $(66 \%)$ showed a decrease in responsiveness, 9 units showed no change, and only 1 unit showed an increased response at low frequencies $(1-10 \mathrm{~Hz})$. Figure 4 shows that the suppression of $\mathrm{S} 1$ cortex significantly reduced the average responsiveness of this sample of $29 \mathrm{VB}$ thalamic neurons at all tested frequencies.

We obtained evidence that the reduction of VB neuronal responsiveness is related to the degree of cortical suppression, as determined by $\mathrm{S} 1$ evoked response amplitude. Evoked response amplitude was reduced to $20 \%$ or less of control values during the study of 17 units, and to $20-50 \%$ while the other 12 units were tested. As is shown in Figure 5, a significant reduction of VB unit responsiveness could be seen only during the more effective periods of cortical response suppression.

\section{Peripheral stimulation}

The responsiveness of VB neurons to stimulation of receptive fields was examined in 16 units, most of whose receptive fields were on the contralateral limbs or trunk. Compared with the responses to medial lemniscal stimulation, the number of spikes elicited by a peripheral stimulus tended to be somewhat higher at stimulus frequencies below $20 \mathrm{~Hz}$ and lower at the higher frequencies, producing a slightly steeper frequency-response function (Fig. 6).

After the $\mathrm{S} 1$ cortex was suppressed by magnesium sulfate alone, 6 units $(38 \%)$ showed a reduction in responsiveness to peripheral stimulation, 9 units showed no change, and 1 unit showed an increased response at high frequencies (see Fig. $7 \mathrm{~B}$ ). The average frequency-response curve of all 16 units before and 


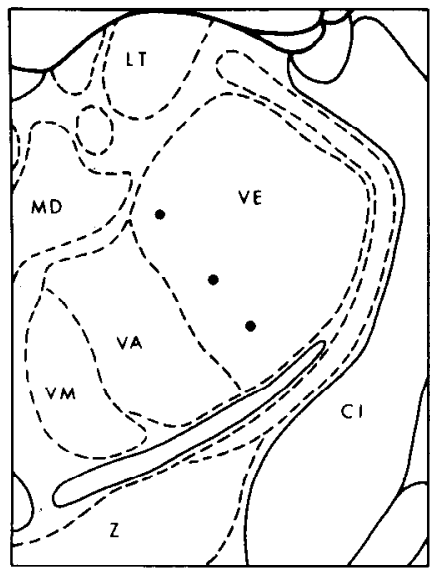

A 5.0

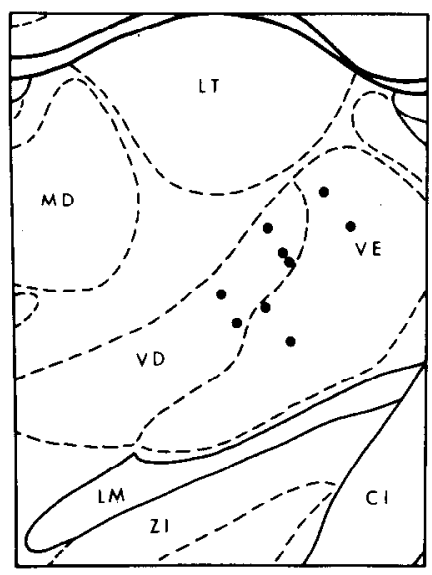

A4.2

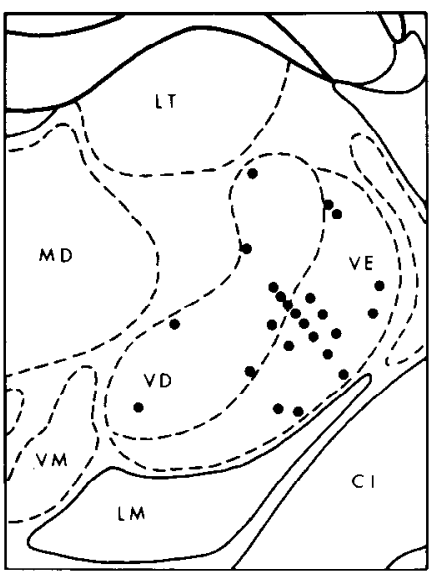

A 4.6

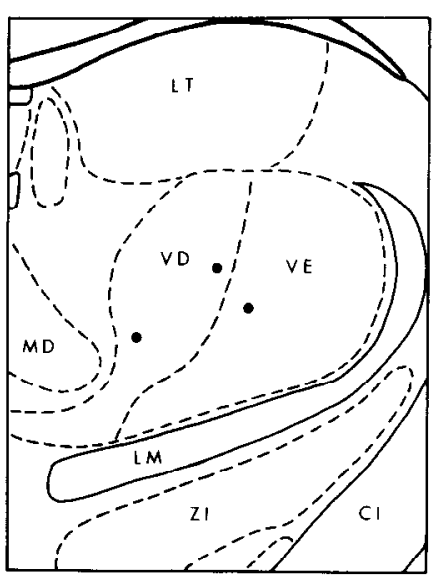

A3.8
Figure 3. Location of recording sites (filled dots) for VB units plotted on rat brain atlas drawings (Pelligrino et al., 1979) at indicated stereouxic AP levels. $C I$, Capsula interna; $L M$, lemniscus medialis; $L T, \mathrm{n}$. lateralis thalami; $M D, \mathrm{n}$. medialis dorsalis; $V A, \mathrm{n}$. ventralis anterior; $V D, \mathrm{n}$. ventralis dorsalis; $V E, \mathrm{n}$. ventralis externa; $V M, \mathrm{n}$. ventralis medialis; $Z I$, zona incerta.

after cortical suppression is shown in Figure 6. Although S1 evoked potentials were reduced to less than $20 \%$ of the control amplitude during testing, the decreased avcrage postsuppression responsiveness seen at low frequencies $(1-20 \mathrm{~Hz})$ was within the $95 \%$ confidence limits of the presuppression average. However, the proportions of peripherally and centrally activated units showing depressed responsiveness were not significantly different $\left(\chi^{2}=2.24, p>0.10\right)$.

\section{Site of cortical influence}

A comparison of the effect of S1 suppression on the responses of the same VB neurons to peripheral and medial lemniscal stimulation should provide information about the site of corticofugal influence on somatosensory transmission. Accordingly, the responsiveness to both peripheral and medial lemniscal stimulation was tested in $6 \mathrm{VB}$ units before and after magnesium sulfate was applied to the $\mathrm{S} 1$ cortex. As is shown in Figure $7 A$, there was a clear reduction in the responses of 5 units to either somatic or medial lemniscal stimulation. A direct comparison of the changes in spikes per stimulus showed no significant difference $(p>0.1 ; t$ test) in the effect of cortical suppression on VB neuronal responses to somatic, as compared with medial lemniscal, stimulation at any stimulus frequency. The remaining unit, which responded to single cutaneous shocks with a burst

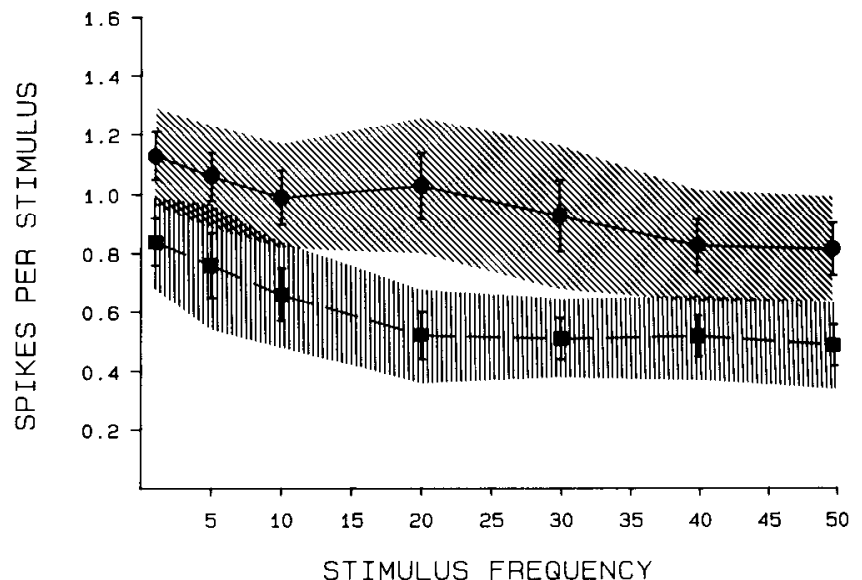

Figure 4. Average ( \pm SEM; $95 \%$ confidence limits) responses of $29 \mathrm{VB}$ units to stimulation of mcdial lemniscus at various frequencies before (filled circles, solid line) and after (filled squares, dashed line) suppression of $\mathrm{S} 1$ cortex in the awake rat.

of 3-4 spikes at both low and high frequencies (Fig. 7B, inset), was unique among all units we recorded in that $\mathrm{S} 1$ cortical suppression produced a modest decrease in responsiveness to 10 $\mathrm{Hz}$ stimuli but a marked increase in the response to cutaneous stimulation at higher frequencies $(20-40 \mathrm{~Hz})($ Fig. $7 B)$.

\section{Other observations}

Other indices of excitability of each of the 39 units were systematically examined. Cortical suppression produced no consistent changes in the frequency or pattern of spontaneous activity, as determined for 38 units (see Materials and Methods). Eighteen units showed decreases ranging from 48 to $90 \%$ (average: $71.4 \%$ ); 17 units showed small increases or decreases, averaging only $2.6 \%$; and only 3 units showed increases $(27,37$, and $309 \%$ ). Changes in spontaneous activity did not parallel changes in responsiveness. Significant or consistent changes in the threshold or latency of the response to peripheral stimulation were not observed. We observed no changes in the size or location of receptive fields following cortical suppression. Although the sample size of units was smaller than that in our previous study, again we could not attribute differences in response to S1 suppression to differences in adequate stimulus (hair, skin, vibrissae) or receptive field location.

\section{Discussion}

The results of this study are consistent with those obtained previously with anesthetized rats (Yuan et al., 1985). Under both experimental conditions, focal suppression of S1 cortical spontaneous activity and evoked responses produced a reduction in the responses of approximately two-thirds of comparably studied VB neurons to repetitive stimulation of the medial lemniscus. The remaining units showed no significant change, and only 1 unit showed partial facilitation. Changes in threshold, latency, spontaneous activity, size, and location of the receptive fields of VB cells were anticipated but not observed following S1 suppression. Although these results are consistent with those of our previous study, the lack of such changes deserves comment because of the results of earlier experiments using electrical stimulation of corticofugal pathways while recording from the dorsal horn or dorsal column nuclei (for a review, see Towe, 1973; Willis and Coggeshall, 1978). In addition to the possibility of species differences, it is likely that focal suppression of $\mathrm{S} 1$ cortex has more limited effects than does electrical stimulation, which can excite recurrent collaterals of thalamocortical neurons or, in the case of pyramidal tract stimulation, activate the axons 


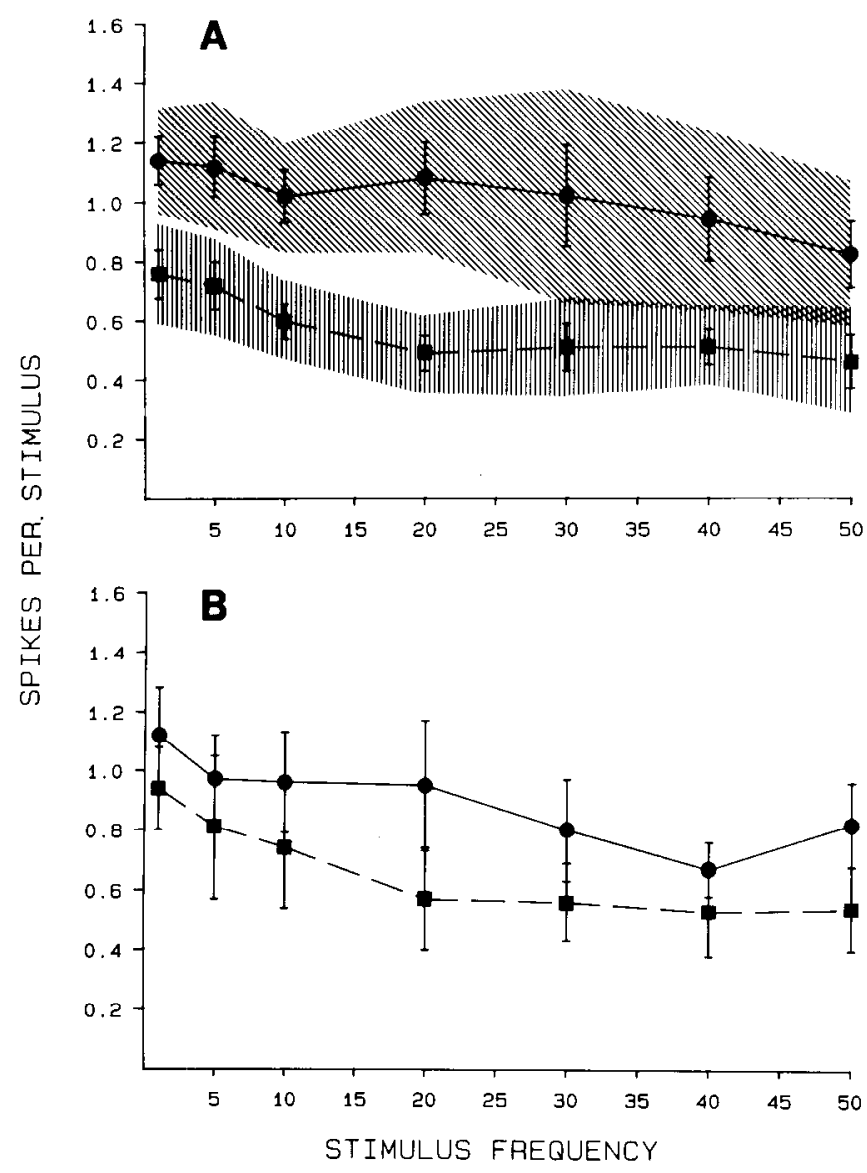

Figure 5. Average $( \pm$ SEM) frequency responses of VB units to medial lemniscal stimulation in the awake rat before and after suppression of S1 evoked response amplitude to $(A) 20 \%$ or less of control amplitude (17 units) or $(B) 20-50 \%$ of control amplitude (12 units). Symbols as in Figure 4. Confidence limits of $95 \%$ are shown in $A$ only; differences shown in $B$ are within the $95 \%$ confidence limits of control.

of neurons outside the S1 cortex (Patton and Amassian, 1960).

Our findings support the interpretation that $\mathrm{S} 1$ corticothalamic influences on VB neurons are predominantly excitatory, as suggested by some other physiological studies (Albe-Fessard et al., 1983; Anderson et al., 1972; Waller and Feldman, 1967) and by evidence that these corticothalamic neurons contain excitatory neurotransmitters (Bromberg et al., 1981; Fonnum et al., 1981; Rustioni et al., 1983). The mechanism of this facilitatory influence cannot be determined on the basis of the available evidence. However, the lack of significant or consistent changes in the spontaneous activity of VB neurons during the period of depressed responsiveness suggests that the facilitatory influence does not act directly on the soma and proximal dendrites of the VB cells from which we recorded.

The major difference between this study and that on anesthetized rats (Yuan et al., 1985) is that, in the present study, S1 suppression failed to produce a significant change in the average responsiveness of 16 units to electrical somatic stimuli. Only 6 $(38 \%)$ of the present sample of units showed response attenuation, whereas $14(70 \%)$ of 20 comparably studied units in the anesthetized rats were similarly affected. The difference between these proportions is not statistically significant $\left(\chi^{2}=2.60, p>\right.$ 0.10 ), but it is nonetheless important to consider why, in the awake rat, corticofugal influences on VB neurons would be more apparent during medial lemniscal, as compared with cutaneous, stimulation. The statistical comparison suggests that a larger sample of cutaneously activated units in the awake rat might

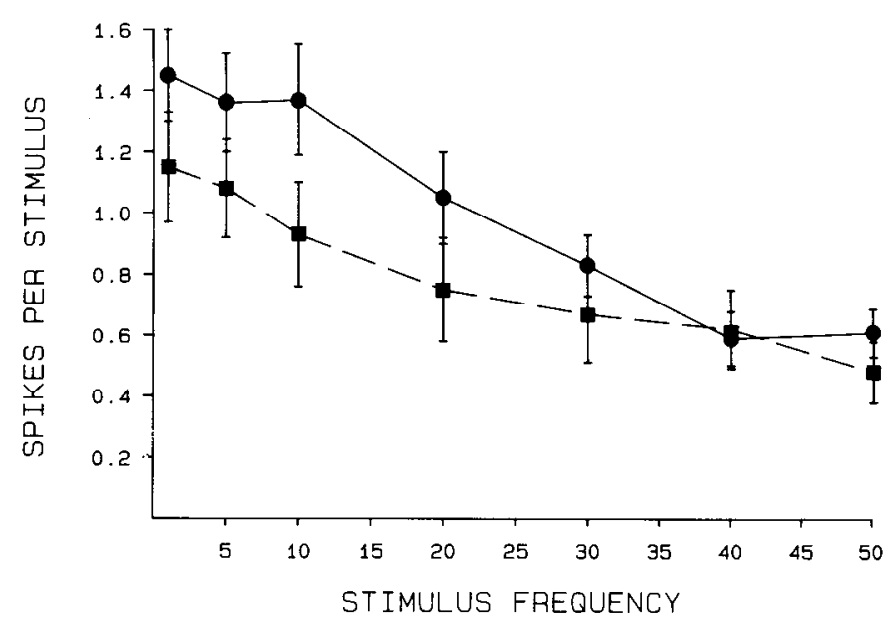

Figure 6. Average ( \pm SEM) responses of $16 \mathrm{VB}$ units to electrical stimulation of the receptive field before and after suppression of $\mathrm{S} 1$ cortex in the awake rat. Differences shown are within $95 \%$ confidence limits of control. Symbols as in Figure 4.

reveal an attenuation of average responsiveness during $\mathrm{S} 1$ suppression. Indeed, the cutaneously activated units show a slightly reduced average response at low stimulus frequencies following S1 suppression, which suggests a trend in the expected direction (Fig. 6). In addition, we found no significant difference in the proportion of peripherally and centrally activated units that showed depressed responsiveness.

It is also possible that the degree of focal suppression of S1 cortex was not sufficient to influence cutaneously activated VB neurons. We were unable to reduce ECoG activity in the awake rat as profoundly as we could in the anesthetized rat because of the convulsant action of lidocaine and the need to maintain the concentration of magnesium sulfate within a range that would not damage the cortex with repeated applications. However, the depressive effect of magnesium alone on spontaneous and evoked cortical neuronal discharge is well known (Kato et al., 1968; Somjen and Kato, 1968) and, in our experiments, was quite effective in nearly eliminating the potential in S1 cortex evoked by medial lemniscal stimulation. We also showed that a reduction in the amplitude of this potential to $20 \%$ or less of control was associated with reduced VB neuronal responses. Furthermore, a degree of $\mathrm{S} 1$ suppression that had no significant effect on the cutaneous responsiveness of VB neurons (Fig. 6) was sufficient to depress VB responses to medial lemniscal stimulation. Fven when the responses of the same VB neurons were compared, the attenuation of responses to medial lemniscal stimuli was apparent, but no additional influence attributable to subthalamic modulation could be seen when cutaneous stimuli were used (Fig. $7 A$ ).

A final possibility is that in the awake, but not the anesthetized, rat there is a corticofugal suppression of subthalamic somatosensory pathways that counterbalances corticothalamic facilitation. If this were true, the effects of $S 1$ suppression would be most apparent when VB unit excitability was tested with medial lemniscal stimulation, because the oppositely affected subthalamic pathways would have been bypassed; the effect of S1 suppression on cutaneously activated VB neurons would be less evident. There is substantial evidence for both inhibitory and excitatory corticofugal modulation of subthalamic somatosensory transmission, but the available evidence provides little basis for favoring one type of influence over another (Towe, 1973; Willis and Coggeshall, 1978). According to our results, the opposing corticofugal influences we have suggested must have converged and counterbalanced one another at or before 
Figure 7. Comparison of changes in the responsiveness (spikes per stimulus) of the same VB units to stimulation of medial lemniscus (filled circles, dashed line) or receptive field (open circles, solid line) following suppression of the $\mathrm{S} I$ cortex in awake rat. $A$, Five units, average \pm SEM. $B$, One unit showing increased excitation to electrical stimulation of the receptive field after suppression of the $\mathrm{S} 1$ cortex. Inset, bursting type of response seen both before and after $\mathrm{S} 1$ cortical suppression.
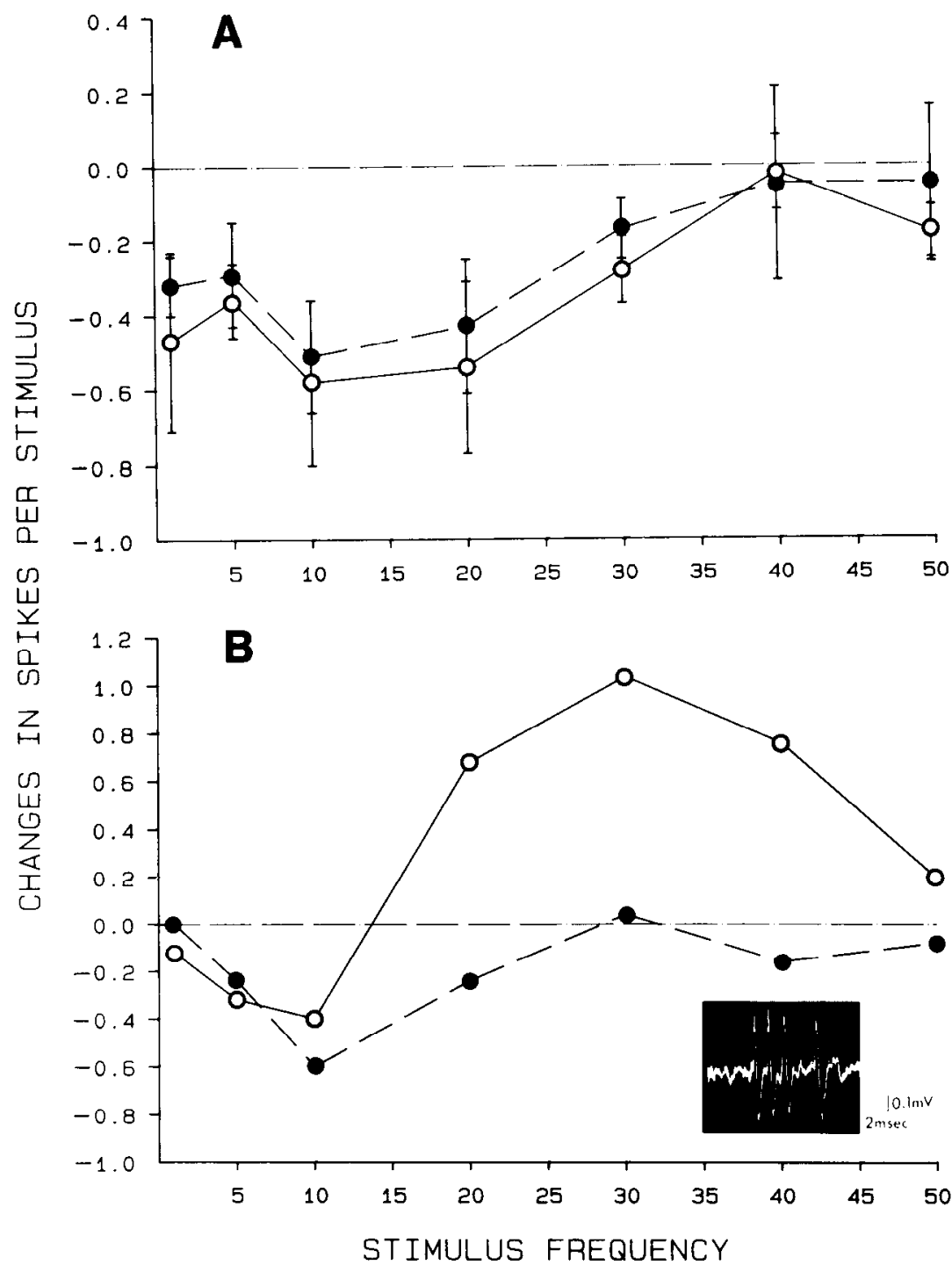

reaching each VB neuron; otherwise, $\mathrm{S} 1$ suppression would produce reduced responsiveness to cutaneous stimuli in one set of VB cells and increased responses in another. Instead, nearly all the VB cells we studied were either unaffected or rendered less responsive to somatic stimuli by $\mathrm{S} 1$ suppression. The single exception was the VB unit showing a clear facilitation of somatic responsiveness during $\mathrm{S} 1$ suppression (Fig. 7B).

Neither our experiments nor the evidence available in the literature provides a firm basis for deciding among the alternatives presented above or among other possibilities not discussed here. Additional experiments will be necessary in order to identify more precisely the effect of general anesthesia on corticofugal controls.

We have recently reviewed some of the relevant literature on cortical control of the VB thalamus (Yuan et al., 1985). Several types of experimental techniques and preparations have been used, and the conclusions reached have often been contradictory. Some of the conflicting results and interpretations can be explained as being due to methodological differences. One such situation arises most prominently when we compare our results with those of Burchfiel and Duffy (1974). They cooled the cortex of unanesthetized cats while recording an immediately subcortical potential evoked by stimulation of the medial lemniscus. The results led them to suggest an inhibitory corticothalam- ic influence, an interpretation conflicting with the results of our experiments on the anesthetized rat (Yuan et al., 1985). On the basis of the present report, it appears that anesthesia does not account for the difference in the conclusions drawn from these 2 studies. It is possible that other methodological differences, such as the use of evoked potential vs unit recording, or the methods used for cortical suppression, could lead to conflicting results and conclusions. It is also likcly that significant variations are due to species differences in VB organization (Ralston, 1983) or to physiological differences between the transient effects on synchronous volleys, as tested by Burchfiel and Duffy (1974) and longer-duration effects on repetitive inputs, as examined in our experiments. However, it is unlikely that we have overlooked major differences between the corticothalamic influence on single, synchronous, as compared with repetitive, afferent, volleys.

Our results show that the effect of S1 suppression is greatest during stimulation frequencies of $10-30 \mathrm{~Hz}$, which indicates a dominant, phasic, evoked corticothalamic facilitation of the repetitive discharge of VB neurons; but our present and previous (Yuan et al., 1985) results also show that there is a tonic corticothalamic facilitation that is sustained for at least $1 \mathrm{sec}$, since the depressive effects of S1 suppression are seen with single afferent volleys delivered at $1 \mathrm{~Hz}$.

Further studies are needed to resolve these and other issues. 
Nonetheless, our experiments provide substantial evidence that somatosensory transmission in the rat VB thalamus is facilitated directly by an excitatory corticothalamic projection to $\mathrm{VB}$, indirectly by the disinhibition of inhibitory thalamic reticular neurons (Ângel, 1983; Houser et al., 1980; Yingling and Skinner, 1976), or by a combination of these mechanisms.

\section{References}

Albe-Fessard, D., M. Condes-Lara, S. Kesar, and P. Sanderson (1983) Tonic cortical controls acting on spontaneous and evoked thalamic activity. In Somatosensory Integration in the Thalamus, G. Macchi, A. Rustioni, and R. Spreafico, eds., pp. 273-285, Elsevier, Amsterdam.

Anderson, P., K. Junge, and O. Sveen (1972) Cortico-fugal facilitation of thalamic transmission. Brain Behav. Evol. 6: 170-184.

Angel, A. (1983) The functional interrelations between the somatosensory cortex and the thalamic reticular nucleus: Their role in the control of information transfer across the specific somatosensory thalamic relay nucleus. In Somatosensory Integration in the Thalamus, G. Macchi, A. Rustioni, and R. Spreafico, eds., pp. 221-239, Elsevier, Amsterdam.

Bromberg, M. D., J. B. Penney, B. S. Stephenson, and A. B. Young (1981) Evidence for glutamate as the neurotransmitter of corticothalamic and corticorubral pathways. Brain Res. 214: 369-374.

Burchfiel, J. L., and F. H. Duffy (1974) Corticofugal influence upon cat thalamic ventrobasal complex. Brain Res. 70:395-411.

Chapin, J. K., and C.-S. Lin (1984) Mapping the body representation in the $S 1$ cortex of anesthetized and awake rats. J. Comp. Ncurol. 229: 199-213.

Fonnum, F., J. Storm-Mathisen, and I. Divac (1981) Biochemical evidence for glutamic neurotransmitter in corticostriatal and corticothalamic fibers in rat brain. Neuroscience 6: 863-873.

Houser, C. R., J. E. Vaughn, R. P. Barber, and E. Roberts (1980) GABA neurons are the major cell type of the nucleus reticularis thalami. Brain Res. 200: 341-354.

Kato, G., J. S. Kelly, K. Krnjevic, and G. Somjen (1968) Anaesthetic action of magnesium ions. Can. Anaesth. Soc. J. 15: 539-544.
Leao, A. A. P. (1944) Spreading depression of activity in the cerebral cortex. J. Neurophysiol. 7: 359-390.

Morrow, T. J. (1980) Improved technique for recording single unit activity in awake animals. Brain Res. Bull. 5:91-93.

Patton, H. D., and V. E. Amassian (1960) The pyramidal tract: Its excitation and functions. In Handbook of Physiology. Neurophysiology II, J. Field, H. W. Magoun, and V. E. Hall, eds., pp. 837-861, Williams and Wilkins, Baltimore, MD.

Pelligrino, L. G., A. S. Pelligrino, and A. J. Cushman (1979) A Stereotaxic Atlas of the Rat Brain, Plenum, New York.

Ralston, H. J., III (1983) The synaptic organization of the ventrobasal thalamus in the rat, cat and monkey. In Somatosensory Integration in the Thalamus, G. Macchi, A. Rustioni, and R. Spreafico, eds., pp. 241-250, Elsevier, Amsterdam.

Rustioni, A., D. E. Schmechel, R. Spreafico, S. Cheema, and M. Cuenod (1983) Excitatory and inhibitory amino acid putative neurotransmitters in the ventral posterior complex: An autoradiographic and immunocytochemical study in rats and cats. In Somatosensory Integration in the Thalamus, G. Macchi, A. Rustioni and R. Spreafico, eds., pp. 365-383, Elsevier, Amsterdam.

Somjen, G. G., and G. Kato (1968) Effects of magnesium and calcium on neurones in the central nervous system. Brain Res. 9: 161-164.

Towe, A. L. (1973) Somatosensory cortex: Descending influences on ascending systems. In Handbook of Sensory Physiology, Vol. II: Somatosensory System, A. Iggo, ed., pp. 701-719, Springer, Berlin.

Waller, H. J., and S. M. Feldman (1967) Somatosensory thalamic neurons: Effects of cortical depression. Science 157: 1074-1077.

Willis, W. D., and R. E. Coggeshall (1978) Sensory Mechanisms of the Spinal Cord, p. 485, Plenum, New York.

Yingling, C. D., and J. E. Skinner (1976) Selective regulation of thalamic sensory relay nuclei by nucleus reticularis thalami. EEG Clin. Neurophysiol. 41: 476-482.

Yuan, B., T. J. Morrow, and K. L. Casey (1985) Responsiveness of ventrobasal thalamic neurons after suppression of $S 1$ cortex in the anesthetized rat. J. Neurosci. 5: 2971-2978. 